\title{
Through the heart and beyond: a review on ranolazine
}

\author{
Carlo Uran
}

Cardiology and Intensive Care Unit, "San Giuseppe e Melorio" Hospital, Santa Maria Capua Vetere (CE), Italy

\begin{abstract}
Ranolazine derives from piperazine and has been approved as a drug for the therapy of chronic stable angina. It acts by selectively inhibiting the late sodium inward current. Moreover, ranolazine has other metabolic features which makes it effective in other diseases as well as coronary artery ones. In this paper I make an updated review of all possible therapeutic roles of ranolazine: through cardiology and beyond.
\end{abstract}

\section{Introduction}

\section{Current use and action mechanisms}

Ranolazine is a derivate of piperazine approved as an antianginal drug for the treatment of patients suffering from stable coronary artery disease, added to other antianginal drugs as atenolol, amlodipine or diltiazem. Ranolazine has not relevant effects on heart rate or blood pressure and inhibits the slowly inactivating component of the cardiac inward sodium current (late iNa), which

Correspondence: Dr. Carlo Uran, Cardiology and Intensive Care Unit, "San Giuseppe e Melorio" Hospital, Via Melorio, 81055 Santa Maria Capua Vetere (CE), Italy. Mobile: +39.339.2683713.

E-mail: carlura@libero.it

Keywords: Ranolazine; chronic stable angina; arrhythmias; diabetes; pulmonary artery hypertension; peripheral arterial disease; non-dystrophic myotonias.

Conflict of interest: The author declares no competing interests and confirms accuracy.

Ethics approval and consent to participate: Not applicable.

Acknowledgements: The author warmly thanks Serena C. Uran for her collaboration in translation.

Received for publication: 15 February 2021.

Accepted for publication: 25 June 2021.

${ }^{\circ}$ Copyright: the Author(s), 2021

Licensee PAGEPress, Italy

Monaldi Archives for Chest Disease 2022; 92:1806

doi: 10.4081/monaldi.2021.1806

This article is distributed under the terms of the Creative Commons Attribution Noncommercial License (by-nc 4.0) which permits any noncommercial use, distribution, and reproduction in any medium, provided the original author(s) and source are credited. reduces the detrimental effects of intracellular sodium and calcium excess that accompany and may cause myocardial ischemia [1]. Experimental studies suggested that ranolazine determines some reduction in fatty acid oxidation with a resultant increasing in glucose oxidation over myocardial stress, which leads to a rising of the amount of ATP synthetized for each oxygen molecule consumed [2]. A recent study by Le et al., in an open chest canine model and cardiomyocytes incubated with ranolazine and with or without adenosine, showed that ranolazine may exert advantageous effects by increasing the activity of the enzyme cytosolic5 '-nucleotidase, which leads to an increasing of adenosine concentration in coronary venous vessels both at rest and during myocardial ischemia induced by dobutamine. Furthermore, the authors demonstrated, in vitro, that ranolazine leads to a rise of adenosine levels in endothelial cells and cardiomyocytes as well. The anti-adrenergic properties of adenosine, and its ability in myocardial protection, are well-identified and its increasing, promoted by ranolazine, may explain the positive effect in ischemic heart diseases [3]. Moreover, there are also evidences that ranolazine has $\alpha 1$-, $\beta 1$-, and $\beta 2$-antiadrenergic activities that have not been completely studied yet [4]. For these mechanisms of action ranolazine may play a therapeutic role in many diseases other than coronary artery ones. In this paper then I make a review all the possible therapeutic roles of ranolazine.

\section{Coronary artery and myocardium diseases}

In 2005, Rousseau demonstrated that patients suffering from chronic angina had better ability to sustain physical exercise, longer time of onset of angina symptoms, and longer time to ST-tract depression in course of therapy with ranolazine versus placebo, with negligible impact both on heart rate and blood pressure. Patients to whom ranolazine was administered were capable to generate more cardiac work than those to whom placebo was. This effect was demonstrated by an increasing of mean RPP (heart rate multiplied by systolic blood pressure) at peak of a physical exercise test. The increasing of cardiac performance with ranolazine versus placebo was not related to a decreasing of cardiac work and of myocardial oxygen consumption. These data were related with an improvement in the myocardium efficiency in using oxygen rather than with a reduced consumption of it [5]. After these findings, the trial MERLIN-TIMI 36 was designed and its results have been published in 2007. This large trial evaluated the effectiveness of the ranolazine in acute coronary syndrome (ACS) without ST segment elevation (NSTEMI). The results of the study are summarized in Table 1. The findings provided also the first demonstration of suppression, by ranolazine, of pro-arrhythmic indicators such as early after-depolarizations (EADs) and transmural dispersion of repolarization (TDR) [6]. These data were consistent with those published in 2004 by Antzelevitch et al. who evaluated the electrophysiological action of 
ranolazine in dog's cardiomyocytes. They showed that ranolazine by inhibiting $\mathrm{iKr}$, late $\mathrm{iNa}$, iCa, late $\mathrm{iCa}$, and $\mathrm{iNa}-\mathrm{Ca}$ fluxes, acts on both inward depolarizing and outward repolarizing ionic currents. In summary, the inhibition of $\mathrm{iKr}$ by ranolazine extends the action potential duration (APD) and the inhibition of late $\mathrm{iNa}$ and late $\mathrm{iCa}$ currents reduces the APD. The result of these actions is a little increasing of the mean QTc interval. Due to this mechanism, the extension of the APD does not pair with EADs, which are responsible of the scattering of repolarization, or polymorphic ventricular tachycardia. Indeed, rather than presenting arrhythmogenic activity, ranolazine, due to its actions in suppressing EADs, and in dropping TDR, conveys a noteworthy antiarrhythmic activity [7]. Ranolazine in the MERLIN TIMI 36 showed a reduction of the risk of relapsing ischemia in patients with non-ST-elevation ACS by probably improving myocardial perfusion. Following this observation, Schwemer et al. [8] assumed that the administration of ranolazine during the first phase of an acute coronary syndrome, by the enhancement of myocardial microcirculation, should probably lead to a recruiting and recovering of hibernating myocardium activity. They published a trial (RIMINI-trial), designed in order to demonstrate that ranolazine, added to standard therapy for six weeks, should be able to reduce the extension of myocardial ischemia in patients suffering from ACS. Twenty patients affected with ACS, were enrolled. To ten of the twenty patients ranolazine was administered, in addition to standard drugs, orally for six weeks and the dose was raised after the first week, from $500 \mathrm{mg}$ bid to $750 \mathrm{mg}$ bid, throughout the study. The extent of the ischemic myocardial area was assessed in all patients by a speckle-tracking echocardiography (SPE), before and after angioplasty. The finding of this study was that patients who were in treatment with ranolazine, had a tendency to higher normal fraction of the global strain than patients who were not. Therefore, adding ranolazine to the standard therapy in patients with ACS, showed effectiveness in decreasing the dyskinetic myocardium area [8]. Some clinical evidences showed the effectiveness of the ranolazine in patients with non-obstructive coronary arteries and in symptomatic patients already undergone to PCI. In 2020 Calcagno et al. evaluated the role of ranolazine in the therapy of 49 consecutive patients with known coronary artery disease, who had already undergone percutaneous myocardial revascularization and who underwent further coronary angiography due to residual angina. The angiography revealed small epicardial coronary vessel $(<2 \mathrm{~mm})$ diseases or non-revascularizable stenosis. The low-dose administration of ranolazine (375 $\mathrm{mg}$ bid), joined to the antiischemic therapy, showed the reduction of exercise angina, increase of exercise tolerance and reduction of exercise arrhythmias [9]. Other clinical evidences showed the effectiveness of ranolazine on coronary vasospasm [10]. In such disease the ranolazine is effective because it increases the levels of adenosine in endothelial cells and by blocking late sodium inward current (iNa) and subsequently it prevents the cytosolic calcium overload, acting as a calcium channel blocker-like [11]. The reduction of intracellular calcium, subsequent to the inhibition of sodium channel, has been verified with pigs, in which ranolazine was administered intracoronary. In this subject ranolazine showed the capability to increase the coronary arteries blood flow and to reduce the resistance of such vessels [12]. A recent work by Rayner-Hartley et al., studied the effectiveness of the ranolazine on 31 patients affected by microvascular angina. The study showed that ranolazine significantly improves symptom control and quality of life in such patients [13]. Inhibition of late iNa relieves diastolic tension as well. In fact, ranolazine acts by mean of a lusitropic effect that decreases the interstitial pressure of myocardium. In the repolarizing ischemic myocardium, late iNa is constantly activated and compels the $\mathrm{Na}^{+}-\mathrm{Ca}^{2+}$ exchanger to work in a opposite manner in order to expel $\mathrm{Na}^{+}$. Because of this action, $\mathrm{Ca}^{2+}$ enters into the myocytes, actives myofilaments and causes diastolic wall tension [14]. In light of this action, Minotti et al. randomized 24 patients to an evaluation of post-chemotherapy (anthracycline) diastolic dysfunction to ranolazine or common cardiovascular drugs. They showed that ranolazine, thanks to its lusitropic effect, is possibly capable to mitigate early diastolic dysfunction, which is a side effect of anticancer drugs [15]. In 2013 the RALI-DHF trial, already evaluated the effectiveness of ranolazine on diastolic disfunction. This study included patients with left ventricular ejection fraction $>45 \%$ and altered diastolic function parameters, who received continuous intravenous infusion of ranolazine for $24 \mathrm{~h}$, followed by oral administration in the following thirteen days. Ranolazine showed to reduce both left ventricular end diastolic and pulmonary capillary wedge pressure [16]. In 2019 Nie et al., showed a more complex

Table 1. The MERLIN-TIMI 36 trial's results.

\begin{tabular}{|c|c|c|c|c|c|}
\hline N. & Patients' characteristics & Dosage & $\mathrm{F} / \mathrm{U}$ & $\mathrm{AE}$ & Results \\
\hline 6560 & $\begin{array}{l}\text { Male and female, average age of } 64 \text { years, } \\
\text { suffering from NSTEMI, with or without } \\
\text { prior MI, affected by one or more of the } \\
\text { following comorbidities: } \\
\text { - Diabetes } \\
\text { - Hypertension } \\
\text { - Hyperlipemia }\end{array}$ & $\begin{array}{l}200 \mathrm{mg} \text { of ranolazine or placebo i.v. over } \\
1 \text { hour, followed } 80 \mathrm{mg} \text { per hour ( } 40 \mathrm{mg} \\
\text { per hour in patients with creatinine } \\
\text { clearance }<30 \mathrm{mg} / \mathrm{ml} \text { ). After } 12-96 \text { hours } \\
\text { of intravenous, oral administration: } \\
\text { ranolazine } 350 \mathrm{mg} \text { bid, } 500 \mathrm{mg} \text { bid or } \\
750 \mathrm{mg} \text { bid, orally, depending on the } \\
\text { clearance of the creatinine clearance }\end{array}$ & 1 year & $\begin{array}{l}\text { Dizziness } \\
\text { Syncope }\end{array}$ & $\begin{array}{l}\text { Ranolazine was not effective in } \\
\text { reducing the rate of the composite } \\
\text { endpoint of cardiovascular death, } \\
\text { myocardial infarction. The study showed } \\
\text { a reduction of recurrent ischemia in } \\
\text { patients with stabilized coronary artery } \\
\text { disease. Furthermore, this large trial } \\
\text { showed the safety of ranolazine. In fact, } \\
\text { there was no increasing of arrhythmias } \\
\text { or sudden cardiac death rate during the } \\
\text { follow-up, in patients receiving } \\
\text { ranolazine compared to those receiving } \\
\text { placebo. The aetiology of the adverse } \\
\text { events was unexplained and not } \\
\text { correlated to arrhythmias. In fact, during } \\
\text { the first seven days, the patient treated } \\
\text { with ranolazine, had a reduction of the } \\
\text { arrhythmias on Holter monitoring }\end{array}$ \\
\hline
\end{tabular}

N, number of patients; NSTEMI, non-ST elevation myocardial infarction; MI, myocardial infarction; F/U, follow up; AE, adverse events. 
action by ranolazine on the calcium metabolic pathway. They studied the effects of ranolazine on both cardiac hypertrophy and heart failure induced by pressure overload in mice-hearts. They observed that the blockade of late inward sodium current arrested the progression towards both cardiac hypertrophy and fibrosis and led to an improvement of cardiac mechanics as well. The authors demonstrated both abnormal $\mathrm{SERCa}^{2+}$ release and activity in hypertrophicinduced ventricular myocytes. The authors demonstrated that ranolazine, by inhibiting the late sodium current, with a subsequent restoring of $\mathrm{Na}^{+}$and $\mathrm{Ca}^{2+}$ normal fluxes, can block the hypertrophic pathways, can mitigate the ER stress and subsequent cardiomyocyte apoptosis (Figure 1) and finally drives to an improvement of both the cardiac hypertrophy induced by pressure overload and the systodiastolic function. Due to these findings, the administration of ranolazine may be a new frontier for the therapy of the cardiac hypertrophy and heart failure [17]. As regards the cardiac hypertrophy, in 2017 Coppini et al. in a mouse-heart model demonstrated that ranolazine, having the capability to reduce the intracellular $\mathrm{Ca}^{2+}$ overload and calmodulin kinase activity, was able to prevent the development of a hypertrophic phenotype in subjects who carried a mutation strongly correlated to the hypertrophic cardiomyopathy (HCM). Given these extremely important findings, ranolazine could be a potential therapy for the prevention of the development of $\mathrm{HCM}$, in young phenotypically negative patients, who carry some mutations strongly correlated to such severe pathology [18]. In 2018 Ferrantini et al. studied the effectiveness of two sodium channels inhibitors (ranolazine and GS-967), in experimentally-induced obstruction. They investigated human myocardium cells, taken from patients suffering from HCM, underwent surgical septal myectomy in order to relieving drug-refractory symptoms related to left ventricular output tract obstruction, under conditions mimicking rest and stress (isoprenaline) [19]. The result of such study is summarized in Table 2. As regards the non-obstructive HCM, in 2018 Olivotto et al., showed that ranolazine although had no great effect on exercise capacity, plasma proBNP levels, diastolic heart failure, or quality of life, was safe and effective in reducing the amount of ventricular extrasystoles, in a cohort of 80 patients [20]. These results could be a consequence of the complexity in the definition of HCM that

Table 2. Effectiveness of two sodium channel inhibitors (ranolazine and GS-967) in experimental-induced left ventricular output tract obstruction by pharmacological stress (isoprenaline).

\begin{tabular}{|c|c|c|c|c|}
\hline Drugs & Subject of study & Control group & Pharmacological stress & Results \\
\hline $\begin{array}{l}\text { - Ranolazine } \\
\text { - GS -967 } \\
\text { us } \\
\text { - Disopyramide } \\
\text { - } \beta \text {-blockers }\end{array}$ & $\begin{array}{l}\text { Human myocardium cells } \\
\text { of } 22 \text { patients undergone } \\
\text { surgical septal myectomy }\end{array}$ & $\begin{array}{l}\text { Myocardium cells from } 5 \text { patients } \\
\text { undergone heart surgery for aortic } \\
\text { valve replacement and septal } \\
\text { myectomy }\end{array}$ & Isoprenaline & $\begin{array}{l}\text { Both ranolazine and GS-967 had } \\
\text { the capability to reduce the } \\
\text { interventricular septal cell stress } \\
\text { during mimicking exercise in vitro } \\
\text { with a certain superiority over } \\
\text { disopyramide and } \beta \text {-blockers. } \\
\text { capability to suppress arrhythmias } \\
\text { induced by isoprenalinepotential } \\
\text { to relief symptoms due to the } \\
\text { obstruction in HCM patients }\end{array}$ \\
\hline
\end{tabular}

HCM, hypertrophic cardiomyopathy.

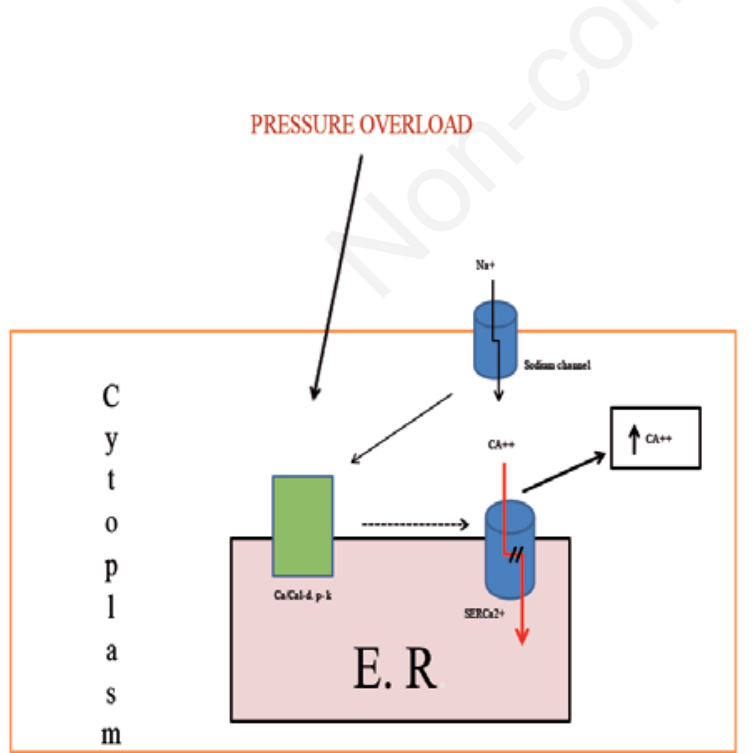

A.

B.

Figure 1. A) Pressure overload actives the calcium/calmodulin-dependent protein kinase which alters the activity of the SERCa2 ${ }^{+}$and plays a fundamental role in cellular proliferation; furthermore, the pressure overload by prolonging endoplasmic reticulum (ER) stress, drove to apoptosis started by ER itself. B) Ranolazine, acting by a complex mechanism, normalized the SERCa2 ${ }^{+}$activity and besides suppressed the calcium/calmodulin-dependent protein kinase. 
includes several characteristics that can somehow hinder the path of a randomized trial [21]. Concerning the heart failure, this study gave an explication to the results of a previous one by Murray et al. who investigated the effect of ranolazine on ejection fraction (EF) in patients with systolic or diastolic disfunction. They showed an increase of left ventricular ejection fraction in such patients when treated with ranolazine. In patients with $\mathrm{HFrEF}$, the EF increased from $30.46 \pm 5.66 \%$ to $36.83 \pm 9.97 \%$, on the other side, in patients with $\mathrm{HFpEF}$, the increasing was from $42.83 \pm 3.46 \%$ to $52.33 \pm 8.59 \%$ [22]. With regards to the heart failure therapy, a brief observation by Marciniak (2019), reported that ranolazine may enhance the collateral effects of ACEIs and ARBs. In light of this, clinicians should monitor for such enhancement when initiating a treatment with ranolazine and an ACEI or ARB [23]. Due to all these findings, ranolazine has been included in the 2019 ESC Guidelines for the diagnosis and management of chronic coronary syndromes, as a second-line treatment to reduce angina frequency and improve exercise tolerance in subjects who cannot tolerate, have contraindications to, or whose symptoms are not adequately controlled by beta-blockers, CCBs, and long-acting nitrates (Class IIa) and, in selected patients, as a first-line treatment according to heart rate, BP, and tolerance (Class IIb) [24]. Lastly, ranolazine has been included in the 2016 ESC Guidelines for the diagnosis and treatment of acute and chronic heart failure and may be considered in patients unable to tolerate a beta-blocker to relieve angina (Class IIb), even if its safety is uncertain [25]. Noteworthy is that this may be considered valid for the heart failure with depressed ejection fraction, but some other evaluations should be done for the treatment of heart failure with preserved ejection fraction, given the results of RALi-DHF study [16] and of the Women's Ischemia Syndrome Evaluation-Coronary Vascular Dysfunction ancillary study [26].

\section{Diabetes}

In spite of medical and interventional advances effective in reducing myocardial ischemia, chronic angina nowadays affects around 8 million people in the United States. Although it is frequently related to a poor quality of life, recurrent hospitalizations, and has a significant impact on health expenditure, angina in often not properly treated. Moreover, patients with both angina and type 2 diabetes mellitus (T2DM) represent a more complex group to treat, since they frequently have multi-vessel coronary artery disease (CAD) in comparison with those without T2DM. In 2013 Kosiborod et al. published the data of TERISA, a randomized, double-blind trial in which ranolazine was tested $v$ s placebo in 949 patients suffering from diabetes, CAD, and stable angina, that was already on treatment with 1-2 antianginals. The trial showed that in patients suffering from diabetes, symptomatic for chronic angina in spite of treatment with two drugs or more, ranolazine was effective in reducing anginal symptoms and sublingual nitroglycerin consumption. Moreover, it was highlighted that treatment with ranolazine, by unclear mechanisms, was more effective in patients with higher HbA1c at baseline. However, it is likely that this beneficial effect could be mediated by the indirect activity of the ranolazine on the calmodulin KII (CaMKII), a kinase that phosphorylating the cardiac sodium channel, causes an increase of intracellular sodium and calcium. Then ranolazine, by inhibiting the sodium current, could have a significant therapeutic effect in patients who present a bad glycemic control. According to an alternative hypothesis, ranolazine may also decrease $\mathrm{HbA} 1 \mathrm{c}$ and such effect is particularly noticeable in patients with uncontrolled dia- betes. The hypothesis of the authors was that, since myocardial perfusion may be worsened by hyperglycemia which has several effects: i) causes microvascular and endothelial dysfunction; ii) increases platelet aggregation; iii) promotes a prothrombotic state; iv) increases the oxidative stress, and besides, since glucose lowering alone has never been shown to improve angina, ranolazine, through inhibition of late $\mathrm{INa}$, should acts against the hyperglycemia effects listed above, resulting to be effective in glucoselowering action [27]. In 2012 Jimenèz-Corona et al. speculated that in insulin resistance, the chronic overexpression of Osteopontin (factor of skeletal muscle inflammatory process) promoted by reactive oxygen species (ROS), may cause chronic inflammation and consequent muscle fibrosis [28]. In 2017, Terruzzi et al., since ranolazine has been shown to be effective in reducing hemoglobin A1c levels in diabetic patients, demonstrated that it could act on mouse skeletal muscle cells regeneration and oxidative process and that due to this action, it could be effective in reducing the insulin resistance.

Due to these observations, all the approaches that lead to a decreasing in mitochondrial dysfunction, ROS levels and inflammatory state, may have therapeutic effectiveness and in the presence of an inflammation process, the effect of ranolazine on osteopontin might lead to reduction of the inflammatory state and IR itself [29] (Figure 2).

On the surface of the pancreatic $\alpha$-cells, voltage-gated $\mathrm{Na}^{+}$ channels are present. Thanks to the electrical activity of such channels, an increase of intracellular calcium is determined, with consequent secretion of glucagon. In 2014 Dhalla et al. in diabetics animal subject, showed, that ranolazine, was able to reduce blood glucagon concentration both in the fasting and in the postprandial period, thus confirming that the effect of ranolazine in glucoselowering is correlated with the blockade of sodium channels. The study pointed out that ranolazine inhibits exocytosis of glucagon from human pancreatic cells and that such inhibition is correlated to the blockade of the tetrodotoxin-sensitive NaChannels, whose isoform is expressed on the surface of the $\alpha$-cells. As a consequence, the administration of ranolazine lowers glucagon and glucose blood levels in diabetic rats [30]. In 2015 Rizzetto et al., hypothesizing that the increasing of sodium current could be one of the mechanisms responsible for insulin deficiency, studied a population of pancreatic $\beta$-cells to evaluate the functional role of the Sodium inward current. The study confirmed that ranolazine, by inhibiting such current, has beneficial effect on insulin secretion. Furthermore, the study confirmed that the ranolazine inhibits the secretion of glucagone from pancreatic $\alpha$-cells as well [31]. In 2018 seven databases were examinated in order to identify all randomized trials comparing the effects of ranolazine versus placebo on hemoglobin A1c, fasting plasma glucose and incidence of hypoglycaemia in patients suffering from chronic stable angina, with or without diabetes. The meta-analytic findings showed that ranolazine can improve glycaemic control in patients with both chronic stable angina and diabetes, and besides can reduce $\mathrm{HbA} 1 \mathrm{c}$ levels, without increased risk of hypoglycemia, both in diabetics and non-diabetics patients [32]. Due to these evidences, ranolazine has been mentioned in the 2019 ESC Guidelines on diabetes, prediabetes, and cardiovascular diseases as second line treatment [33]. Ranolazine may have possible effectiveness as a drug against the diabetic neuropathy too. Diabetic neuropathy is a frequent complication in about $50 \%$ of diabetic patients and the first clinical manifestation is the sensory neuropathy, otherwise autonomic and motor ones occur later [34]. The pathogenesis of diabetic neuropathy appears to be due to a combination of various factors: i) hyperglycaemia; ii) activation of polyol pathway; iii) microangiopathy 
of vasa nervorum; iv) oxidative stress, and lastly v) neuroinflammation [35]. With regards to the painful diabetic neuropathy, at least two other mechanisms seem to be involvesd: variations in the sodium and calcium channels, and central pain modulation systems [36]. In sperimental models of neurons and glia cells, ranolazine has shown to protect those cells. This protective effect by ranolazine is due to the increasing of the of PPAR- $\gamma$ protein production and to the decreased production of tumor necrosis factoralpha (TNF- $\alpha$ ) and interleukin (IL)-1 $\beta$ one in those cells [37]. Furthermore, ranolazine has shown the capability to attenuate neuronal reactivity in sodium channels-mediated neuropathic pain [38]. Pioglitazone, a drug used in type- 2 diabetes, is a PPAR- $\gamma$ agonist [39]. It suppresses hyperalgesia in diabetic neuropathy, by reducing the stimulation of spinal nociceptive neurons [40] and prevents the onset or the pregression of neuropathic pain, acting as an inflammation modulator [41]. These considerations made Elkholy et al. to compare the effects of ranolazine versus pioglitazone on experimentally induced diabetic neuropathy. Their study showed that the beneficial effects of Pioglitazone on diabetic neuropathy, are correlated to the inhibition of the of pro-inflammatory cytokines, which are down regulated both centrally and periferically. Particularly pioglitazone acts by blocking the production of IL$6, \mathrm{TNF}-\alpha$ in cell cultures of astrocyte and microglia. In the injured peripheral nerve, pioglitazone has shown the ability to reduce hyperalgesia dy decreasing the expression of inflammatory cytokines and the influx of macrophages. This benefical actions
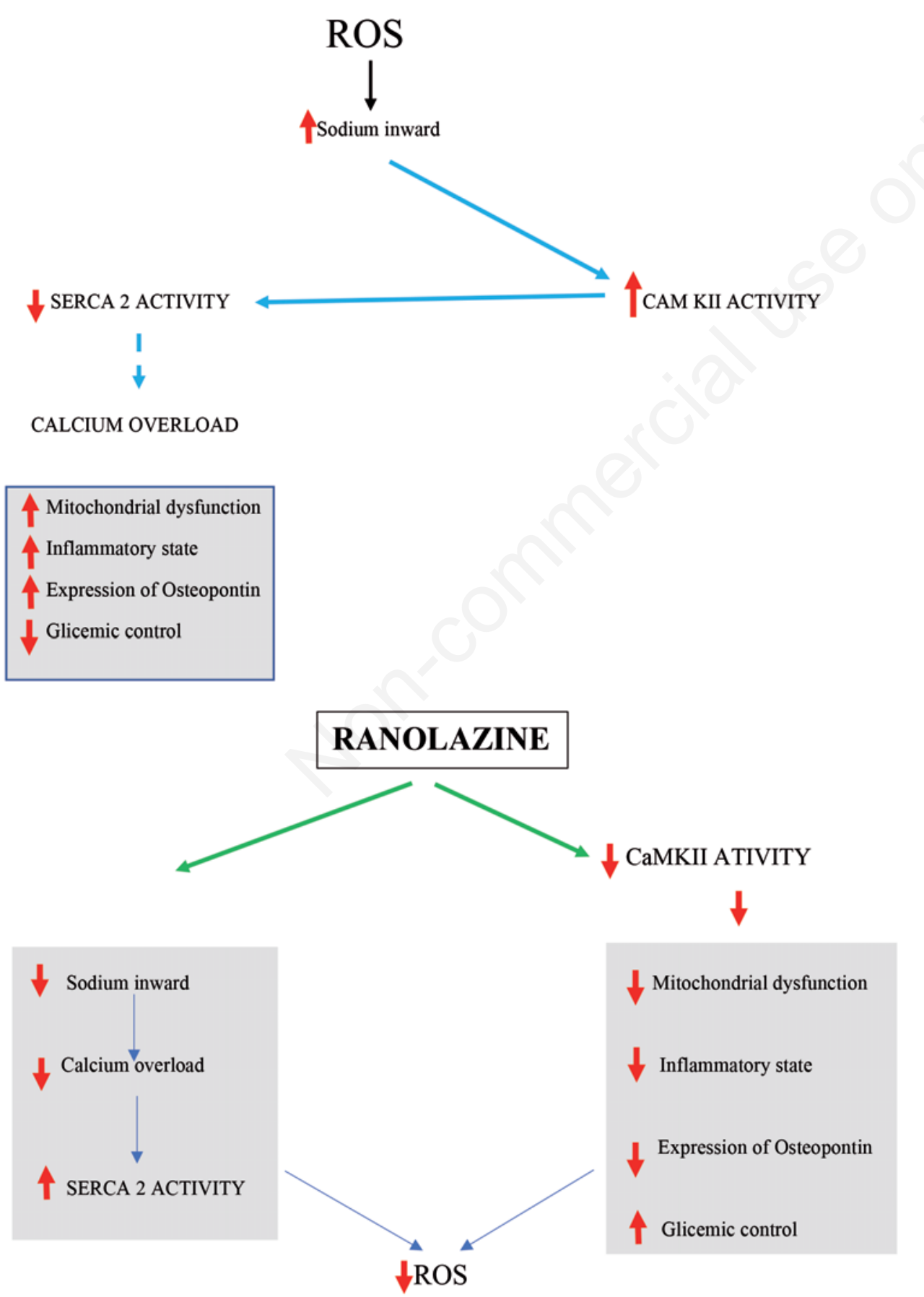

Figure 2. Effect of the ROS increase and of the ranolazine. ROS may cause chronic inflammation. Ranolazine through phosphorylation may reduce the CaMKII activity and might control glucose metabolism. Besides, the inhibition of late iNa and the subsequent lowering in intracellular calcium, leads to a reduction of ROS. Mitochondria are a wellknown source of ROS and the ranolazine has the capability in decreasing the number of mitochondria in proliferation and differentiation stages consequently demonstrated its antioxidant role. Noteworthy, in skeletal muscle cells, increasing of oxidative stress leads to insulin resistance. Both the combination of inflammatory cascades and the activation of kinases following the oxidative stress, may be among the causes that contribute to altering the response to insulin secretion. This is the evidence that oxidative stress plays a fundamental role in the inflammatory response and is responsible for mitochondrial damage and for the induction of insulin resistance in skeletal muscle cells. 
are enhanced by the activation of PPAR $\gamma$ with the consequent increasing of production of proteins that inhibit the inflammatory process and promote the repair of the injured nerve itself. The authors showed that ranolazine was able to reduce the production of TNF- $\alpha$ and IL- $1 \beta$ at the peripheral nerve level and furthermore, to increase the PPAR- $\gamma$ expression as well. The explanation for such an effect could be that ranolazine, by blocking the sodium channel, could reduce the IL- $1 \beta$ and TNF- $\alpha$ and then the IL1 $\beta$ expression. This experimental work showed that ranolazine has neuroprotective effects comparable to pioglitazone [42]. Lastly, type 2 diabetes mellitus is an important risk factor for cognitive impairment [43]. Recently, Cassano et al. demonstrated that ranolazine has effects against cognitive impairment and depressive-like behavior, in rats with type 2 diabetes. This property is absolutely new and non completely clarified concerning the molecular mechanisms involved. If further studies prove the effectiveness of ranolazine against such diseases, it will be another step forward for the treatment of diabetes complications [44].

\section{Peripheral arterial disease}

Sodium channels are not only in myocardium cells but also in skeletal muscle cells and have both a fast and slow phase. This is the reason why the sodium channels on the skeletal muscle cells can respond to ischemia in a similar way as the miocardium ones do. Furthermore, it must be remembered that ranolazine also improves endothelial function. In fact, in an animal model, has been shown that intra-femoral artery injection of the drug determines a lasting dilation of the artery itself, comparable to that obtained with nitroglycerin. This effect may be due to the $\alpha 1-$ adrenergic receptor blockade, which does not determine alterations of both the heart rate and the systemic blood pressure [12]. In 2006 a pilot study by Ma et al., involving 45 patients with chronic obstructive arteriopathy of lower limbs suffering from intermittent claudication, ranolazine $1000 \mathrm{mg}$ bid showed an improvement of the walking time of approximatively $40 \%$ without an increase of the Winsor index, displaying a better effect than placebo, and comparable to cilostazol. This result, although concerned a pretty small patients' sample, has stimulated further research [45]. The results of a randomized trial comparing the effects of ranolazine versus placebo in supervised treadmill exercise, will be soon published [46]. Pending these results, ranolazine is not mentioned as therapy to add to aspirin or clopidogrel or oral anticoagulation in the 2017 ESC/ESVS Guidelines on the Diagnosis and Treatment of Peripheral Arterial Diseases [47], neither in 2019 European Society for Vascular Medicine Guideline on peripheral arterial disease [48].

\section{Pulmonary artery hypertension}

Pulmonary arterial hypertension (PAH) is a progressive and a poor prognosis disease, in which are present vasoconstriction, endothelial dysfunction, smooth muscle cell proliferation, and thrombosis of pulmonary arterioles, that lead to the right ventricular failure [49]. The hemodynamic features of the disease are mean pulmonary arterial pressure equal or greater than $25 \mathrm{~mm} \mathrm{Hg}$ at right heart catheterization, with pulmonary arterial occlusion pressure equal or less than $15 \mathrm{~mm} \mathrm{Hg}$ and pulmonary vascular resistance above 3 Wood units. The most recent clinical classification of pulmonary hypertension by European Society of Cardiology (ESC) and European Respiratory Society (ERS) identifies five pulmonary hypertension $(\mathrm{PH})$ groups and each of them contains several subgroups. The group 1 includes idiopathic, heritable $\mathrm{PH}$ and those associated with systemic diseases (connective tissue disease) and secondary to pulmonary veno-occlusive disease; the group 2 includes PH caused by left heart disease; the group 3 includes PH caused by lung disease and/or hypoxia; the group 4 includes chronic thromboembolic PH and other pulmonary artery obstructions; e the group 5 includes $\mathrm{PH}$ with unclear and/or multifactorial mechanisms. The assignment of a patient suffering from to one of the five groups and subgroups, is fundamental for his management [50]. Despite the presence of several therapies targeting the pulmonary vascular bed, there are currently no therapies that are specifically aimed for the treatment of right ventricular (RV) failure in patient suffering from PAH. Noteworthy is that RV dysfunction is one of the major determinants of the poor outcomes in patients suffering from PAH [51]. Recently, Yuchi has studied the effect on RV function, in patients with pre-capillary $\mathrm{PH}$, of 6-months treatment with ranolazine, using cardiac magnetic resonance imaging. They enrolled 22 patients, 15 of them completed the study: 9 were in the ranolazine group, and 6 in the placebo group. Ranolazine in precapillary $\mathrm{PH}$ patients on treatment with $\mathrm{PH}$-specific vasodilators demonstrated to improve RV function, LV end-diastolic volume, and biventricular stroke volumes. The authors concluded that it is effective in improving RV function in patients suffering with precapillary $\mathrm{PH}$ [52]. The mechanism by which ranolazine improves $\mathrm{RV}$ function is not well clarified, but the most likely hypothesis is that, in patients with significant $\mathrm{PAH}$, the presence of RV ischemia is very common when the disease passes from a phase of compensation to a phase of worsening. The RV ischemia progresses to RV failure. The coronary flow of the RV differs from the LV one. In fact, the blood flow through the left coronary artery occurs mainly during diastole, while the flow through the right coronary artery occurs during the entire heart cycle. This happens because the aortic pressure is higher than the pulmonary one, both during systole and diastole. In patients with PAH there is an increasing of the RV systolic pressure which becomes similar to the aortic one and the pressure gradient between the aorta and the RV results nullified, so that it may receive blood from the right coronary artery exclusively during the diastolic phase. As a consequence of this process, the $\mathrm{RV}$, which has extremely thin wall, becomes progressively ischemic and the increasing of the end-diastolic pressure, which prevents coronary flow even during diastole, contributes to this. The increasing of the heart rate and the reduction of the duration of diastole during exercise, make it worse. Ranolazine, by blocking the late iNa, prevents calcium overload, responsible for diastolic dysfunction, in myocardial cells. In this way the diastolic tension of the RV is reduced and the blood flow to the ischemic RV itself during diastole is improved. As a final result ranolazine improves RV performance and contractility [53].

\section{Arrhythmias}

Ranolazine is structurally correlated to the lidocaine, a class $\mathrm{Ib}$ antiarrhythmic drug which is safe and effective in the control of the ventricular arrythmias induced by ischemia. It is known that the lidocaine has no effect on atrial arrythmias [54]. In the 2020 ESC Guidelines for Atrial Fibrillation, class Ic and class III antiarrhythmic drugs are recommended in order to maintain sinus rhythm in patients suffering from recurrent atrial fibrillation [55]. In 2015 the 
RAFFAELLO Trial enrolled 238 patients with persistent atrial fibrillation (AF) to whom, after effective electrical cardioversion, instead of a class I or III antiarrhythmic drug, ranolazine, at doses of 375,500 , or $750 \mathrm{mg}$ twice daily for 16 weeks, was prescribed. The aim of the study was to demonstrate if ranolazine was effective in maintaining the sinus rhythm. The trial failed in its primary end point of time to AF recurrence. However, despite this result, the study suggested a possible role for the high-dose ranolazine in the prevention of arrhythmia [56]. Again in 2015 the study HARMONY evaluated the effectiveness of ranolazine and dronedarone in reducing the AF burden in patients with an implanted pacemaker. At the baseline the AF burden was $17.4 \%$ (range 2\%-72\%), and placebo or dronedarone $225 \mathrm{mg}$ twice daily, were not effective in significantly reduce the AF burden. In contrast, the administration of ranolazine $750 \mathrm{mg}$ twice daily, dronedarone $150 \mathrm{mg}+$ ranolazine $750 \mathrm{mg}$ bid, and dronedarone $225 \mathrm{mg}+$ ranolazine 750 $\mathrm{mg}$ bid, proved effective in reducing the AF burden by $23.0 \%$, $45.5 \%$, and $59.1 \%$ from baseline [57]. Previously, Koskinas et al. underwent pharmacological cardioversion 121 patients with recent-onset $\mathrm{AF}$. They administered intravenous amiodarone alone $(5 \mathrm{mg} / \mathrm{kg}$ in $60 \mathrm{~min}$ followed by $50 \mathrm{mg} / \mathrm{h}$ until the sinus rhythm was restored or a maximum for $24 \mathrm{~h}$ ) or in combination with ranolazine $1500 \mathrm{mg}$ once, at the same intravenous regimen. Few patients were converted to sinus rhythm by amiodarone alone, unlike what happened with cordarone plus ranolazine combined therapy $(70 \%$ vs $87 \%, \mathrm{p}=0.024)$. Moreover, the cardioversion time turned out to be longer in patients treated with Amiorarone alone, than in those treated with the two drugs in cooperation $(13.3 \pm 4.1 \mathrm{~h}$ vs $10.2 \pm 3.3 \mathrm{~h}, \mathrm{p}=0.001$ ) [58]. In $2018 \mathrm{De}$ Vecchis et al. published a metanalysis in which concluded that ranolazine, at adeguate doses, is effective in reducing the conversion time of AF when combined with amiodarone, compared to amiodarone alone. Moreover, in patients with sinus rhythm, ranolazine is able to reduce the frequency of the new-onset AF and its recurrence, especially in patients undergone coronary artery bypass grafting (postoperative AF) [59]. In 2020 Wolfes and al. investigated, in a rabbits' hearts model, the effects of ranolazine in combination with different selective inhibitors of the $\mathrm{Na}^{+} / \mathrm{Ca}^{2+}$-exchanger (ORM10103 and SEA0400) on AF induced by infusion of isoproterenol (Iso) and acetylcholine (ACh). The infusion of Iso/ACh resulted in a shortening of the duration of the atrial action potential, of the effective atrial refractory period and of the monophasic action potential, so that the recurrence of AF increased significantly. The perfusion with ranolazine and ORM-10103 by significantly prolonging the refractory periods and monophasic action potential, was able to reduce the episodes of AF. On the other side, the association between ranolazine and SEA0400 determined a slight decreasing in $\mathrm{aAPD}_{90}$ and a prolonging in aERP and aPRR so that the occurrence of AF episodes was consecutively reduced [60]. Functionally the ranolazine can be compared to the class Ia or III of antiarrhythmic drugs, since, just like them, it acts blocking the rapid component of the delayed rectifier potassium current (IKr). The blocking of this ion current, just as class III antiarrhythmics do, results in the phase 3 variation in the action potential of myocardial cells, that is in fact increased, resulting in an extension of the actual atrial refractory period. In addition, blocking the sodium channels, such as class I antiarrhythmics, affects both peak and late sodium current which play an important role during the phase 0 and 2 of the cardiac potential of action, respectively. Furthermore, the blockade of the inward Sodium peak, leads to an increase of the post-repolarisation refractoriness and of the effective refractory period too. At therapeutic concentrations, ranolazine blocks very significantly the late iNa. Such blockade reduces the L-type inward calcium current and therefore the triggers to the onset of AF, such as re-entry or early and delayed after depolarizations [61] (Figure 3). Despite the obvious similarities, it is also rationally associate ranolazine with other antiarrhythmic drugs (i.e., amiodarone or dronedarone) since it blocks the sodium channels in a different state than amiodarone and dronedarone do. In fact, ranolazine blocks the channel in the open state, unlike amiodarone and dronedarone which block it in the open one. With regards to class Ia drugs such quinidine and disopyramide, they block the channel in both active and open state and slowly dissociate from the channel itself, so that the risk of torsade de pointes $(\mathrm{TdP})$ is increased, unlike amiodarone and dronedarone who quickly dissociate. On the other hand, regarding ventricular muscle cells instead, the $\mathrm{iKr}$ causes an increase in APD by preventing potassium from leaving the cells. Ranolazine attenuates this effect by blocking the sodium channel and so prevents the sodium from entering in the cells. The result is a small increase in the QTc interval that is balanced by the reduction in transmural dispersion of repolarization because, as ranolazine prolongs APD in epicardial cells but on the other hand, reduces it in the myocardial ones and in the Purkinje fibres. In light of the above, drugs such as ranolazine and amiodarone, although prolonging the QTc interval, have a low risk of TdP. Furthermore, in disease such heart failure or myocardial hypertrophy, where APD is prolonged per se, ranolazine is useful in shortening the whole APD and the QTc interval. Thanks to these evidences, the ranolazine can be used as an additional therapy in AF when other therapeutic options are contraindicated due to the risk of TdP. Due to its mechanisms of action, ranolazine has been demonstrated its effectiveness in ventricular arrhythmias too. In Long QT-2 syndrome, the ranolazine has demonstrated the capability to prevent the calcium overload by stabilizing and desensitizing the ryanodine receptors, with the result that early afterdepolarizations and TdP are suppressed [62]. Moreover, the ranolazine, in experimental models of LQT3 syndrome, carrying the D1790G mutation of the SCN5A, causes a concentration-dependent blockade of iNaL, without significantly reducing the peak iNa. This evidence suggests that the ranolazine may be safe in LQT3 patients, who are at risk of developing Brugada syndrome if treated with sodium channels blockers. In fact, in a small register of 8 patients with LQT3 syndrome, it was observed that the effect of the ranolazine in shortening the QT tract, also persists during night bradycardia. This finding is of extreme clinical relevance, since in patients with LQT3 syndrome, sudden death occurs especially at night [63]. With regard to ventricular arrhythmias, it has been observed that the ranolazine, at therapeutic concentrations in humans, manages to suppress both re-entrant and multifocal VF and that the simultaneous administration of ranolazine and dronedarone, is effective in protecting both against susceptibility to induced-ischemia AF and ventricular arrhythmias [64,65]. Even in experimental models of heart failure, the ranolazine has been shown to retain antiarrhythmic properties. In fact, it prevents the induction of VF by producing post-repolarisation refractoriness [66]. Since the mechanical stretch, which occurs in cases of pressure overload or contractile desynchrony, is a great arrhythmogenic mechanism, in an experimental study the ranolazine showed to reduce the acceleration and the complexity of VF produced by the stretched myocardium [67]. At last recently, in a rabbit model, Moschovidis et al. showed that the ranolazine appears to have a dose-dependent beneficial effect on re-entry paced-induced ventricular arrhythmia during the late phase of myocardial infarction. In this respect, it has been shown that the higher dose of ranolazine has antiarrhythmic effects, while the lower one seems to be ineffective or even proarrhythmic. The authors supposed that the mecha- 
nism by which the ranolazine exerts its beneficial effects could be related to a significant increase in ventricular refractoriness possibly secondary to the IKr blockade [68].

\section{Myotonia and paramyotonia congenita}

Channelopathies, particularly those affecting the sodium channel, may also affect che skeletal muscle as well as the myocardium. Among the 9 known sodium channels, the NaV1.4 one, which is not significantly present in other tissues, is mainly expressed in the skeletal muscle cells, where it is responsible for most of the cellular inward of sodium, which generates the action potential.
Mutations that affect the gene encoding such channel led to skeletal muscle diseases such as paramyotonia congenita and myotonic dystrophy. On the other hand, the myotonia congenita is a skeletal muscle disease caused by a mutation of the chloride channel, is characterized by muscle hyperexcitability and has a negative impact on the quality of life. However, its peculiarity is the socalled warm-up phenomenon, whereby the muscle stiffens tends to improve with exercise. The paramyotonia congenita has an autosomal dominant inheritance and unlike paramyotonia congenita, it worsens with exercise and is extremely cold sensitive. This phenomenon is exactly opposite to the warm-up one [69]. The warmup phenomenon that characterizes the $\mathrm{MC}$, is due to a slow inactivation of sodium channel and ranolazine by promoting such inactivation, may be effective in the therapy of these patients. In a

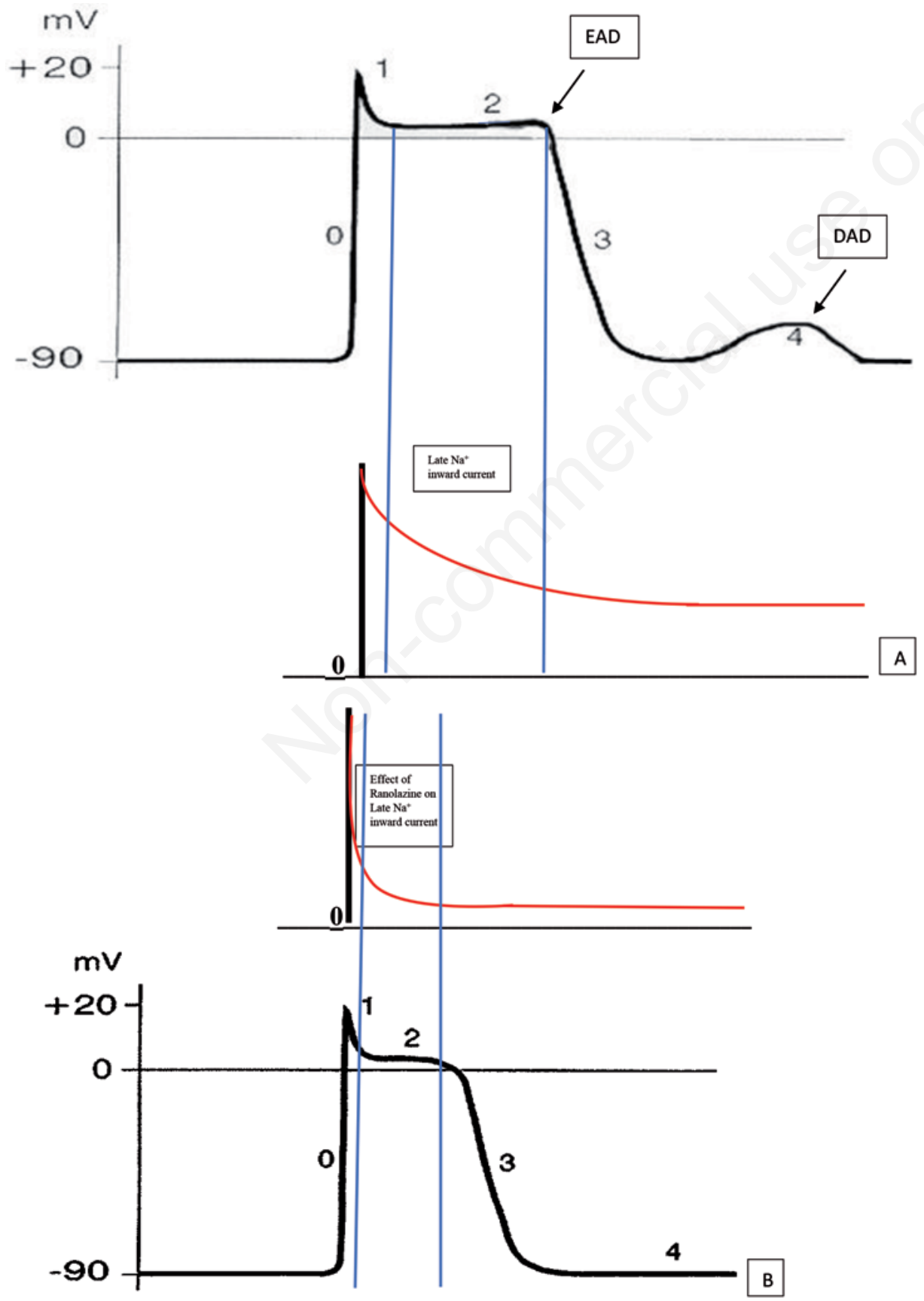

Figure 3. Abnormal myocardial action potential (A); effect of ranolazine $(B)$. The ranolazine by inhibiting of late sodium inward current, shorts the phase 2 of the action potential and suppresses the early depolarization (EAD) and the delayed repolarization (DAD). 'PHASE 0': OVERSHOOT. The potential rapidly increases to +20 $\mathrm{mV}$ due to the peak of $\mathrm{Na}+$ inward current. 'PHASE 1': INITIAL RIPOLARIZATION. The potential has a little decrease due to the $\mathrm{K}^{+} / \mathrm{Cl}^{-}$outward current. 'PHASE 2': PLATEAU. Both the late $\mathrm{Na}^{+}$ and the $\mathrm{Ca}^{++}$inward currents equal the $\mathrm{K}^{+}$outward one. 'PHASE 3: FINAL RIPOLARIZATION. The $\mathrm{Ca++}$ channels are closed; the $\mathrm{K}^{+}$ outward current goes on. 'PHASE 4': RESTING. The activity of the $\mathrm{Na} / \mathrm{K}$ ATPase expels $3 \mathrm{Na}^{+}$from the cell for $2 \mathrm{~K}^{+}$. At the same time both a $\mathrm{Na}^{+} / \mathrm{Ca}^{++}$exchanger and a $\mathrm{Ca} / \mathrm{Na}$ ATPase expel $\mathrm{Ca}^{++}$from the cell. 
recent pilot study involving 13 patients, ranolazine has been shown to improve the symptoms of mioclonia congenita, in terms of clinical and electromyography assessment. Due to these findings, ranolazine may serve as an alternative to mexiletine [70]. Recently, Lorusso et al. evaluated the effectiveness of ranolazine in paramyotonia Congenita and showed that ranolazine significantly improved both subjective symptoms and clinical myotonia [71]. Due to these evidences, the ranolazine has been mentioned in the 2020 Guidelines on clinical presentation and management of nondystrophic myotonias [72].

\section{Conclusions}

Ranolazine, approved as an antianginal agent for the treatment of patients suffering with stable coronary artery disease, added to a standard dose of others antianginal drugs, it acts by selectively inhibiting the late sodium inward current (late iNa). It reduces the detrimental effects of intracellular sodium and calcium excess that accompanies and may cause myocardial ischemia itself. Due to this mechanism of action, ranolazine is effective, other than in stable coronary artery disease, also in biventricular heart failure, in glycaemic control and in myoclonia congenita and paramyoclonia. Due to its versatility the ranolazine owns therapeutic possibility probably not fully investigated.

\section{References}

1. Chaitman BR. Ranolazine for the treatment of chronic angina and potential use in other cardiovascular conditions. Circulation 2006;113:2462-72.

2. Clarke B, Wyatt KM, McCormack JG. Ranolazine increases active pyruvate dehydrogenase in perfused normoxic rat hearts: evidence for an indirect mechanism. J Mol Cell Cardiol 1996;28:341-50.

3. Le DE, Davis CM, Wei K, et al. Ranolazine may exert its beneficial effects by increasing myocardial adenosine levels. Am J Physiol Heart CircPhysiol 2020;318:H189-H202.

4. Zhao G, Walsh E, Shryock JC, et al. Antiadrenergic and hemodynamic effects of ranolazine in conscious dogs. J Cardiovasc Pharmacol 2011;57:639-647

5. Rousseau MF, Pouleur H, Cocco G, Wolff AA. Comparative efficacy of ranolazine versus atenolol for chronic angina pectoris. Am J Cardiol 2005;95:311-6.

6. Morrow DA, Scirica BM, Karwatowska-Prokopczuk E, et al. Effects of ranolazine on recurrent cardiovascular events in patients with non-ST-elevation acute coronary syndromes: the MERLIN-TIMI 36 randomized trial. JAMA 2007;297:1775-83.

7. Antzelevitch C, Belardinelli L, Zygmunt AC, et al. Electrophysiological effects of ranolazine, a novel antianginal agent with antiarrhythmic properties. Circulation 2004; 110:904-10

8. Schwemer TF, Radziwolek L, Deutscher N, et al. Effect of ranolazine on ischemic myocardium in patients with acute cardiac ischemia (RIMINI-Trial): A randomized controlled pilot trial. J Cardiovasc Pharmacol Ther 2019;24:62-9.

9. Calcagno S, Infusino F, Salvi N, et al. The role of ranolazine for the treatment of residual angina beyond the percutaneous coronary revascularization. J Clin Med 2020;9:2110.

10. Uran C, Di Chiara G, Bosco B, et al. A case of vasospastic angina. Vasospasm physiopathology: a new therapeutic role for ranolazine? Monaldi Arch Chest Dis 2020;90:1295.

11. Ghosh GC, Ghosh RK, Bandyopadhyay D, et al. Ranolazine: Multifaceted role beyond coronary artery disease, a recent perspective. Heart Views 2018;19:88-98.

12. Nieminen T, Tavares CAM, Pegler JRM, et al. Ranolazine injection into coronary or femoral arteries exerts marked, transient regional vasodilation without systemic hypotension in an intact porcine model. Circ Cardiovasc Interv 2011;4:481-7.

13. Rayner-Hartley E, Parvand M, Humphries KH, et al. Ranolazine for symptomatic management of microvascular angina. Am J Ther 2020;27:e151-8.

14. Stone PH. Ranolazine: new paradigm for management of myocardial ischemia, myocardial dysfunction, and arrhythmias. Cardiol Clin 2008;26:603-14.

15. Minotti G, Menna P, Calabrese V, et al. Pharmacology of ranolazine versus common cardiovascular drugs in patients with early diastolic dysfunction induced by anthracyclines or nonanthracycline chemotherapeutics: A phase $2 \mathrm{~b}$ minitrial. J Pharmacol Exp Ther 2019;370:197-205.

16. Maier LS, Layug B, Karwatowska-Prokopczuk E, et al. RAnoLazIne for the treatment of diastolic heart failure in patients with preserved ejection fraction: The RALI-DHF proof-of-concept study. JACC Heart Fail 2013;1:115-22.

17. Nie J, Duan Q, He M, et al. Ranolazine prevents pressure overload-induced cardiac hypertrophy and heart failure by restoring aberrant $\mathrm{Na}+$ and $\mathrm{Ca} 2+$ handling. J Cell Physiol 2019;234:11587-601.

18. Coppini R, Mazzoni L, Ferrantini C, et al. Ranolazine prevents phenotype development in a mouse model of hypertrophic cardiomyopathy. Circ Heart Fail 2017;10:e003565.

19. Ferrantini C, Pioner JM, Mazzoni L, et al. Late sodium current inhibitors to treat exercise-induced obstruction in hypertrophic cardiomyopathy: an in vitro study in human myocardium. Br J Pharmacol 2018;175:2635-52.

20. Olivotto I, Camici PG, Merlini PA, et al. Efficacy of ranolazine in patients with symptomatic hypertrophic cardiomyopathy: The RESTYLE-HCM randomized, double-blind, placebo-controlled study. Circ Heart Fail 2018;11:e004124.

21. Elliott PM. Evolving story of clinical trials in hypertrophic cardiomyopathy. Circ Heart Fail 2018;11:e004572.

22. Murray GL, Colombo J. Ranolazine preserves and improves left ventricular ejection fraction and autonomic measures when added to guideline-driven therapy in chronic heart failure. Heart Int 2014;9:66-73.

23. Knuuti J, Wijns W, Saraste A, et al. 2019 ESC Guidelines for the diagnosis and management of chronic coronary syndromes. Eur Heart J 2020;41:407-7.

24. Marciniak TA, Serebruany V. Ranolazine, ACE inhibitors, and angiotensin receptor blockers. Am J Med 2019;132:e844-5.

25. Ponikowski P, Voors AA, Anker SD, et al. 2016 ESC Guidelines for the diagnosis and treatment of acute and chronic heart failure: The Task Force for the diagnosis and treatment of acute and chronic heart failure of the European Society of Cardiology (ESC)Developed with the special contribution of the Heart Failure Association (HFA) of the ESC. Eur Heart J 2016;37:2129-2200.

26. Rambarat CA, Elgendy IY, Handberg EM, et al. Late sodium channel blockade improves angina and myocardial perfusion in patients with severe coronary microvascular dysfunction: Women's Ischemia Syndrome Evaluation-Coronary Vascular Dysfunction ancillary study. Int J Cardiol 2019;276:8-13.

27. Kosiborod M, Arnold SV, Spertus JA, et al. Evaluation of 
ranolazine in patients with type 2 diabetes mellitus and chronic stable angina. Results from the TERISA randomized clinical trial. J Am Coll Cardiol 2013;61:2038-45.

28. Jiménez-Corona AE, Damián-Zamacona S, Pérez-Torres A, et al. Osteopontin upregulation in atherogenesis is associated with cellular oxidative stress triggered by the activation of scavenger receptors. Arch Med Res 2012;43:102-11.

29. Terruzzi I, Montesano A, Senesi P, et al. Ranolazine promotes muscle differentiation and reduces oxidative stress in $\mathrm{C} 2 \mathrm{C} 12$ skeletal muscle cells. Endocrine 2017;58:33-45.

30. Dhalla AK, Yang M, Ning Y, et al. Blockade of Na+ channels in pancreatic $\alpha$-cells has antidiabetic effects. Diabetes 2014;63:3545-56.

31. Rizzetto R, Rocchetti M, Sala L, et al. Late sodium current (INaL) in pancreatic $\beta$-cells. Pflugers Arch 2015;467:1757-68.

32. Teoh IH, Banerjee M. Effect of ranolazine on glycaemia in adults with and without diabetes: a metaanalysis of randomised controlled trials. Open Heart 2018;5:e000706.

33. Cosentino F, Grant PJ, Aboyans V, et al. 2019 ESC Guidelines on diabetes, pre-diabetes, and cardiovascular diseases developed in collaboration with the EASD. Eur Heart J 2020;41:255-323.

34. Iqbal Z, Azmi S, Yadav R, et al. Diabetic peripheral neuropathy: epidemiology, diagnosis, and pharmacotherapy. Clin Ther 2018;40:828-49.

35. Vikram A, Tripathi DN, Kumar A, Singh S. Oxidative stress and inflammation in diabetic complications. Int $\mathrm{J}$ Endocrinol 2014;2014:679754.

36. Feldman EL, Nave K-A, Jensen TS, Bennett DLH. New horizons in diabetic neuropathy: mechanisms, bioenergetics, and pain. Neuron 2017;93:1296-313.

37. Aldasoro M, Guerra-Ojeda S, Aguirre-Rueda D, et al. Valles, Effects of ranolazine on astrocytes and neurons in primary culture. PLoS One 2016;11:e0150619.

38. Gould H, Garrett C, Donahue RR, et al. Ranolazine attenuates behavioral signs of neuropathic pain. Behav Pharmacol 2009;20:755-8.

39. Ding S, Shen Z, Chen Y, et al. Pioglitazone can ameliorate insulin resistance in low-dose streptozotocin and high sucrosefat diet induced obese rats. Acta Pharmacol Sin 2005;26:575-80.

40. Griggs RB, Donahue RR, Adkins BG, et al. Pioglitazone inhibits the development of hyperalgesia and sensitization of spinal nociresponsive neurons in type 2 diabetes. J Pain 2016;17:359-73.

41. Yonutas HM, Sullivan PG, Targeting PPAR isoforms following CNS injury. Curr Drug Targets 2013;14:733-42.

42. Elkholy SE, Elaidy SM, El-Sherbeeny NA, et al. Neuroprotective effects of ranolazine versus pioglitazone in experimental diabetic neuropathy: Targeting Nav1.7 channels and PPAR- $\gamma$. Life Sci 2020;250:117557.

43. Geijselaers SLC, Sep SJS, Stehouwer CDA, Biessels GJ. Glucose regulation, cognition, and brain MRI in type 2 diabetes: A systematic review. Lancet Diabetes Endocrinol 2015;3:75-89.

44. Cassano V, Leo A, Tallarico M, et al. Metabolic and cognitive effects of ranolazine in type 2 diabetes mellitus: Data from an in vivo model. Nutrients 2020;12:382.

45. Ma A, Garland WT, Smith WB, et al. A pilot study of ranolazine in patients with intermittent claudication. Int Angiol 2006;25:361-9.

46. ClinicalTrials.gov [Internet]. Bethesda: National Library of Medicine. Supervised treadmill exercise and ranolazine for intermittent claudication of lower extremities (STERILE). 2009
Jun 4 - Identifier NCT00914316. Available from: Available from: https://www.clinicaltrials.gov/ct2/show/NCT00914316

47. Aboyans V, Ricco JB, Bartelink MEL, et al. 2017 ESC Guidelines on the Diagnosis and Treatment of Peripheral Arterial Diseases, in collaboration with the European Society for Vascular Surgery (ESVS). Eur Heart J 2018;39:763-816.

48. Frank U, Nikol S, Belch J, et al. ESVM Guideline on peripheral arterial disease. Vasa 2019;48:1-79.

49. Tuder RM, Archer SL, Dorfmüller P, et al. Relevant issues in the pathology and pathobiology of pulmonary hypertension. J Am Coll Cardiol 2013;62:d4-D12.

50. Galie N, Humbert M, Vachiery JL, et al. 2015 ESC/ ERS guidelines for the diagnosis and treatment of pulmonary hypertension: the Joint Task Force for the Diagnosis and Treatment of Pulmonary Hypertension of the European Society of Cardiology (ESC) and the European Respiratory Society (ERS): endorsed by: Association for European Paediatric and Congenital Cardiology (AEPC). International Society for Heart and Lung Transplantation (ISHLT). Eur Respir J 2015;46:903-75.

51. van de Veerdonk MC, Kind T, Marcus JT, et al. Progressive right ventricular dysfunction in patients with pulmonary arterial hypertension responding to therapy. J Am Coll Cardiol 2011;58:2511-9.

52. Han Y, Forfia P, Vaidya A, et al. Ranolazine improves right ventricular function in patients with precapillary pulmonary hypertension: results from a double blinded randomized placebo-controlled trial. J Cardiac Fail 2021;27:253-7.

53. Khan SS, Cuttica MJ, Beussink-Nelson L, et al. Effects of ranolazine on exercise capacity, right ventricular indices, and hemodynamic characteristics in pulmonary arterial hypertension: a pilot study. Pulm Circ 2015;5:547-56.

54. Rosa GM, Dorighi U, Ferrero S, et al. Ranolazine for the treatment of atrial fibrillation. Expert Opin Investig Drugs 2015;24:825-36.

55. Hindricks G, Potpara T, Dagres N, et al. 2020 ESC Guidelines for the diagnosis and management of atrial fibrillation developed in collaboration with the European Association of CardioThoracic Surgery (EACTS). Eur Heart J 2021;42:373-498.

56. De Ferrari GM, Maier LS, Mont L, et al. Ranolazine in the treatment of atrial fibrillation: results of the dose-ranging RAFFAELLO (Ranolazine in Atrial Fibrillation Following an ELectricaL CardiOversion) study. Heart Rhythm 2015;12:872-8.

57. Reiffel JA, Camm AJ, Belardinelli L, et al. The HARMONY trial: combined ranolazine and dronedarone in the management of paroxysmal atrial fibrillation: mechanistic and therapeutic synergism. Circ Arhythm Electrophysiol 2015;8:1048-56.

58. Koskinas KC, Fragakis N, Katritsis D, et al. Ranolazine enhances the efficacy of amiodarone for conversion of recentonset atrial fibrillation. Europace 2014;16:973-979.

59. De Vecchis R, Ariano C, Giasi A, Cioppa C. Antiarrhythmic effects of ranolazine used both alone for prevention of atrial fibrillation and as an add-on to intravenous amiodarone for its pharmacological cardioversion: a meta-analysis. Minerva Cardioangiol 2018;66:349-59.

60. Wolfes J, Ellermann C, Broer N, et al. Antiarrhythmic effect of ranolazine in combination with selective NCX-inhibition in an experimental model of atrial fibrillation. Pharmaceuticals (Basel) 2020;13:321.

61. Gupta T, Khera S, Kolte D, et al. Antiarrhythmic properties of ranolazine: a review of the current evidence. Int J Cardiol 2015;187:66-74.

62. Parikh A, Mantravadi R, Kozhevnikov D, et al. Ranolazine sta- 
bilizes cardiac ryanodine receptors: a novel mechanism for the suppression of early afterdepolarization and torsades de pointes in long QT type 2. Heart Rhythm 2012;9:953-60.

63. Chorin E, Hu D, Antzelevitch C, et al. Ranolazine for congenital long-QT syndrome type III: experimental and long-term clinical data. Circ Arrhythm Electrophysiol 2016;9:e004370.

64. Morita N, Lee JH, Xie Y, et al. Suppression of re-entrant and multifocal ventricular fibrillation by the late sodium current blocker ranolazine. J Am Coll Cardiol 2011;57:366-75.

65. Verrier RL, Pagotto VP, Kanas AF, et al. Low doses of ranolazine and dronedarone in combination exert potent protection against atrial fibrillation and vulnerability to ventricular arrhythmias during acute myocardial ischemia. Heart Rhythm 2013;10:121-7.

66. Frommeyer G, Schmidt M, Clauss C, et al. Further insights into the underlying electrophysiological mechanisms for reduction of atrial fibrillation by ranolazine in an experimental model of chronic heart failure. Eur J Heart Fail 2012;14:1322-31.

67. Chorro FJ, del Canto I, Brines L, et al. Ranolazine attenuates the electrophysiological effects of myocardial stretch in Langendorff-perfused rabbit hearts. Cardiovasc Drugs Ther 2015;29:231-41.

68. Moschovidis V, Simopoulos V, Stravela S, et al. Dose-dependent effects of ranolazine on reentrant ventricular arrhythmias induced after subacute myocardial infarction in rabbits. J Cardiovasc Pharmacol Ther 2020;25:65-71.

69. Cannon SC. Channelopathies of skeletal muscle excitability. Compr Physiol 2015;5:761-90.

70. Arnold WD, Kline D, Sanderson A, et al. Open-label trial of ranolazine for the treatment of myotonia congenita. Neurology 2017;89:710-3.

71. Lorusso S, Kline D, Bartlett A, et al. Open-label trial of ranolazine for the treatment of paramyotonia congenita. Muscle Nerve 2019;59:240-3.

72. Stunnenberg BC, LoRusso S, Arnold WD, et al. Guidelines on clinical presentation and management of nondystrophic myotonias. Muscle Nerve 2020;62:430-44. 